\title{
STUDIES ON PAIN. OBSERVATIONS ON PAIN DUE TO LOCAL COOLING AND ON FACTORS INVOLVED IN THE “COLD PRESSOR" EFFECT
}

\author{
By STEWART WOLF AND JAMES D. HARDY \\ (From the Russell Sage Institute of Pathology, in affiliation with the New York Hospital and \\ the Departments of Medicine and Psychiatry, Cornell University \\ Medical College, New York City)
}

(Received for publication May 6, 1941)

When a subject's hand is immersed in cold water two phenomena are known to occur. The subject experiences pain and an elevation of blood pressure. The latter phenomenon has been used as the basis for the Hines-Brown "cold pressor" test (1). The occurrence of pain has received little attention, and is alluded to only incidentally in the articles on the "cold pressor" test. Pain, however, has been suspected of being concerned in the production of the hypertension (2). In the present work on pain due to local cooling, we have found direct correlation between the degree of cooling, the intensity of pain induced by the cold, and the height to which the blood pressure rises.

Lewis (3), in an analysis of the vascular reaction to cold, noted that in fingers immersed in cold water from $0^{\circ} \mathrm{C}$. to $18^{\circ} \mathrm{C}$. a painful aching occurred "soon" after immersion. No attempt was made to study the nature of the pain. The burning pain produced on the skin of the arm by a dry ice stimulator has been studied (4). The pain was shown to be adaptable and, contrary to Goldscheider's theory that pain is an exaggerated pressure sensation, it was shown to be a separate sensory function. The mechanism of production of the pain, however, was not investigated.

The aim of this communication is to report on a study of the phenomenon of pain due to local cooling, which, for convenience, we call " cold pain". The pain is conveniently produced and, owing to its predictable character, lends itself to analysis.

\section{METHOD}

The subject was placed in a reclining position on a cot beside an earthenware crock holding 20 liters of water which was stirred vigorously by a propeller attached to a small motor. Because of the large volume, the bath could be maintained at constant temperature for the duration of an experimental observation without the aid of a thermostatic device. The left hand of the subject was plunged into the water up to the wrist. The time of onset of pain was recorded and an attempt was made to estimate the intensity of pain being suffered at a given moment.

While these observations were being made, the blood pressure in the opposite arm was estimated by means of a mercury sphygmomanometer at as frequent intervals as possible. The skin temperature of the immersed hand was determined continuously by the use of a copper and constantan thermocouple fixed to the pad of the terminal phalanx of the middle finger. The amplitude of pulsations of the digital artery was also recorded by using a small glass plethysmograph fitted to the index finger of the immersed hand. The plethysmograph was connected by an air system to a tambour on which was mounted a small mirror. Movements of the latter were recorded on a camera by reflecting a beam of light on the moving film. Extraneous factors which are known to influence the amplitude of pulsation of the digital artery (5 to 13) were all carefully controlled.

The results of a series of 54 experiments which provide 121 observations are reported. The observations were made mainly by the authors upon one another, since uninterested subjects could scarcely be called upon to undergo the necessary amount of physical discomfort.

\section{OBSERVATIONS}

\section{Sensations induced by local cooling}

A. Deep aching pain and its "adaptation". Immersing the hand in water warmer than $18^{\circ} \mathrm{C}$. caused no pain, but at $18^{\circ} \mathrm{C}$., and slightly below, there was a fleeting deep ache which occurred after the hand had been immersed about $60 \mathrm{sec}-$ onds, and then promptly ceased. At progressively lower temperatures the pain had its onset sooner and was more intense, always reaching its maximum at about 1 minute. It then began to subside, and in 4 to 5 minutes was no longer perceived. The character of the pain was aching; there was a feeling as if the hand had been crushed. The distribution was generalized and deep throughout the immersed hand, and the pain was perhaps most intense on the radial side. It was continu- 
ous and non-pulsatile. Exercise of the fingers or movement of the hand did not influence the character or intensity of the pain. There was no tenderness in the hand since it could be struck against the side of the bath without altering the sensation of pain. Sixty seconds after immersion the peak of pain intensity was reached. This was followed by amelioration, and finally cessation of the pain. The disappearance of pain while the hand is still immersed in the water we have called "adaptation".

A barely perceptible painful sensation experienced as a deep ache in the immersed hand was designated as threshold pain. Additional increments in pain intensity were indicated in terms of pulses, $8+$ being used for the pain of greatest intensity experienced. The distribution of pain as high as $2+$ was confined to the hand. At
$3+$, however, there occurred a radiation of the ache up the inner aspect of the arm. At $4+$ pain was felt in the axilla. At this intensity the subjects usually showed a reaction to the stimulus characterized by rapid irregular respirations, as well as by adventitious movements of the feet. At $5+$ the restlessness was replaced by writhing movements and the subject's face betrayed suffering. From $6+$ to $8+$ the pain provoked perspiration and approached the unbearable level. During the first minute after immersing the hand in cold water it was quite clear that the pain was becoming more and more severe. Each time a definite increase in pain intensity was felt the subject called out an additional plus. Similarly, as adaptation occurred after one minute and the pain was clearly becoming less intense, the subject called out one less plus at each perceptible de-

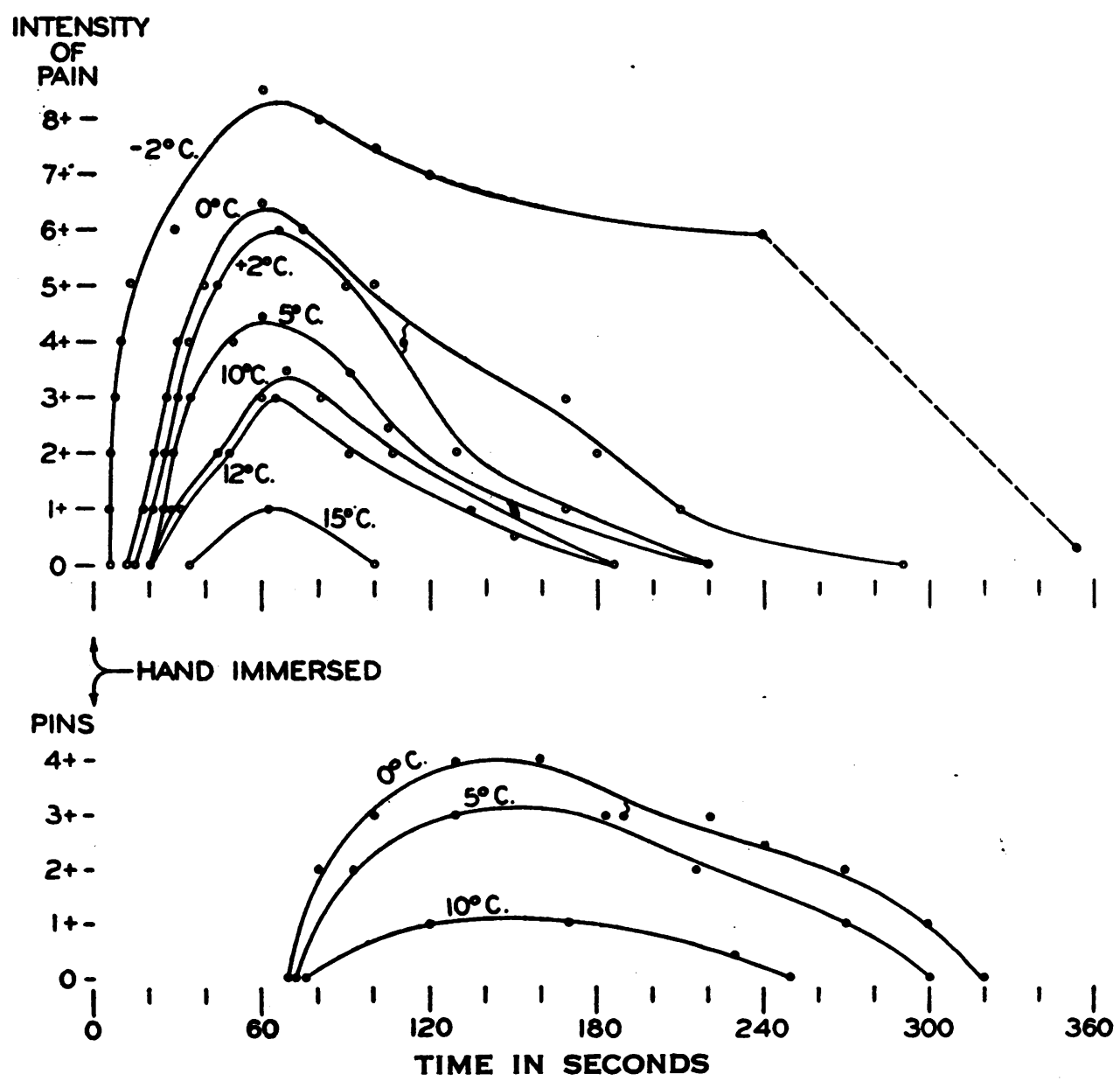

Fig. 1. The Course of the Estimated Intensity of Pain and "Pins and Needles" Sensation Experienced With Various Strengths of Cold Stimulus 


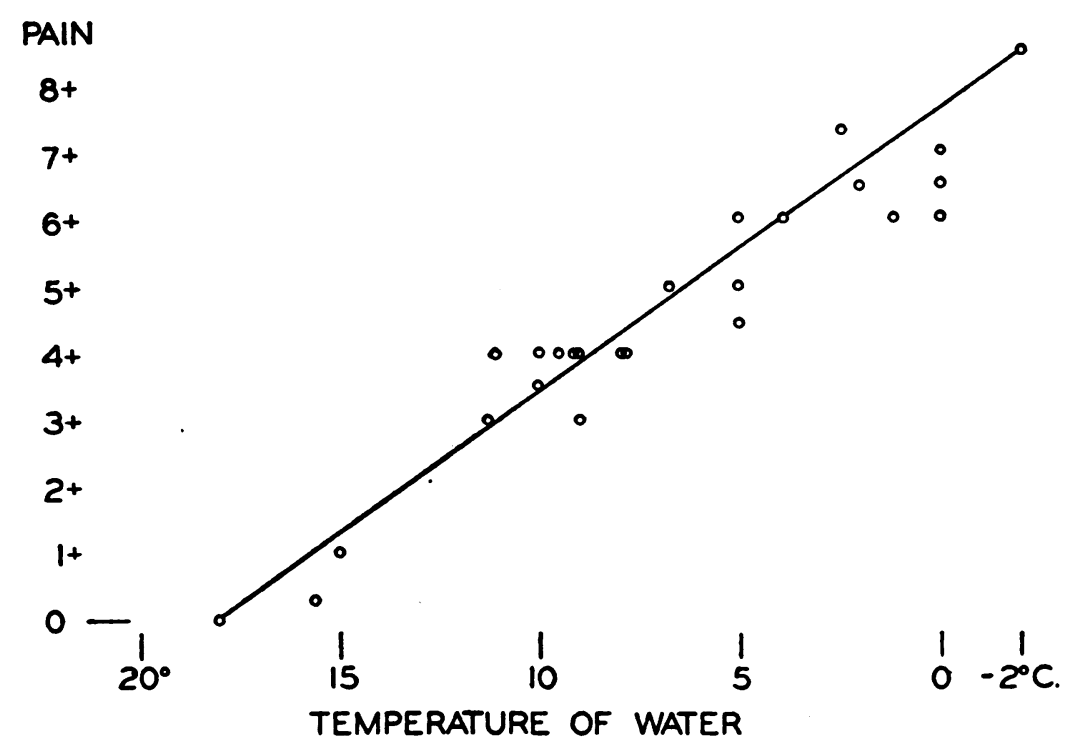

Fig. 2a. The Estimated Peak of Pain Intensity Plotted Against the Strength of the Cold Stimulus

crease in pain intensity. The values thus established were roughly reproducible at any time. A subject immersing his hand in water of unknown temperature consistently called out the same number of pluses as had been previously assigned to that particular temperature. Therefore, although not precise, this method has proved useful here as in other studies of sensation $(14,15)$. Figure 1 shows the course of estimated pain intensity in the hand immersed in water at different temperatures. Figure 2a shows maximal pain intensity plotted against water temperature. It is apparent that pain intensity as estimated increases uniformly with the strength of stimulus measured in terms of temperature of the water bath. It is also of interest that the line points directly at $18^{\circ} \mathrm{C}$. which, as noted above, was found to be the highest temperature at which this variety of pain occurs. In Figure $2 \mathrm{~b}$ the total area under the pain curves at various temperatures is plotted against the temperature of the bath. The total amount of pain experienced also increases uniformly with the strength of stimulus.

$B$. "Pins and Needles" sensation. The sensation of "pins and needles" occurred only in water colder than $12^{\circ} \mathrm{C}$. The sensation was felt in the entire immersed hand from 60 to 90 seconds after immersion. This sensation, occurring shortly after the peak of the aching pain, steadily increased in intensity as the pain decreased. The

\section{AREA BENEATH \\ PAIN CURVE \\ SQ UNITS}

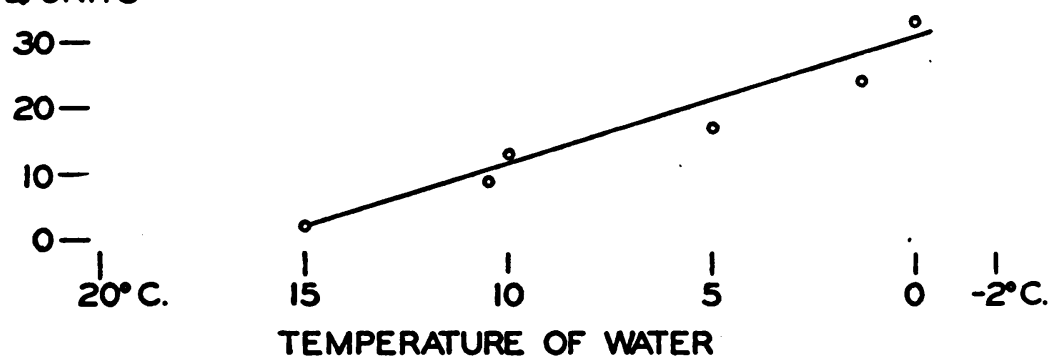

Fig. 2b. The Total Area Beneath the Curves of Pain Intensity in Figure 1 Plotred Against Strength of Cold Strmulus 
lower the bath temperature, the more intense was the prickling. Like pain, this sensation ran a cycle of aggravation and then adaptation, until at the end of 8 to 10 minutes the immersed hand felt only the cold of the water (Figure 1). The sensation of touch and of pain to pin prick, however, as well as motor power in the fingers, remained intact. This "pins and needles" sensation, when intense, caused a great deal of discomfort to the subject. At $-2^{\circ}$ C., although the aching pain had been tolerated well past its peak of severity, the subject was forced to withdraw his hand owing to the suffering imposed by the growing intensity of the "pins and needles" sensation.
C. "Adaptation". If the hand were withdrawn before the pain had reached its maximum intensity, there was a momentary twinge of extra pain immediately after withdrawal. If the hand were withdrawn at any time before complete "adaptation" had taken place and then at once re-immersed, the pain would recur with nearly as great an intensity as occurred with the original immersion. If, however, the hand were left in until "adaptation" had taken place, it could be withdrawn and re-immersed in the cold water without pain being experienced (Figure 3 ). This adaptive effect which protected the hand from pain diminished as the skin temperature was allowed to

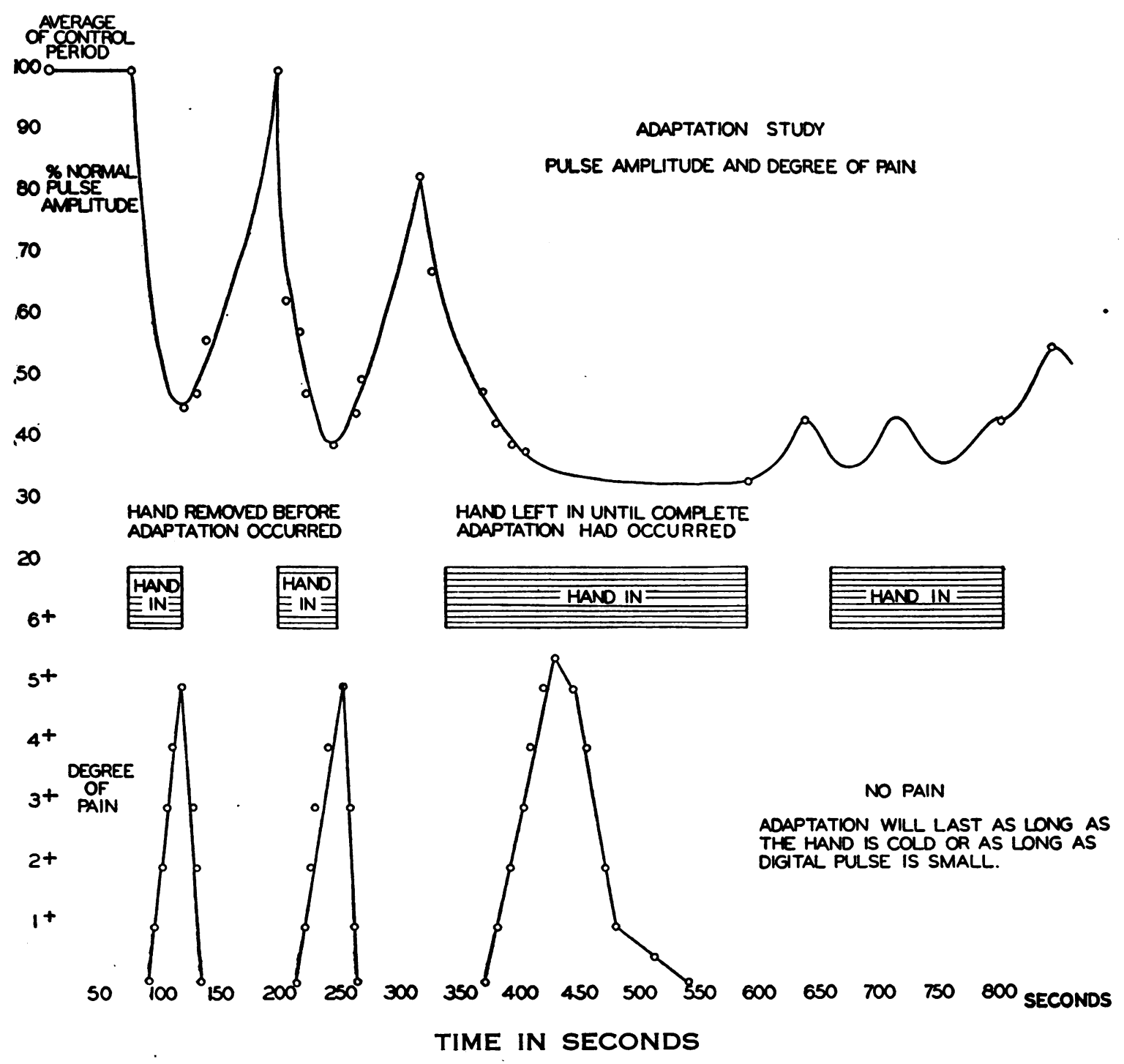

Fig. 3. Study of adaptation. The Relationship Between the Temporal Course of Pain Sensation. its "Adaptations" and the Amplitude of Pulsation of the Digital Artery 
rise. There was partial protection, however, until the temperature of the hand reached $33^{\circ} \mathrm{C}$. At this point, an immersed hand would feel the usual intensity of pain regardless of how quickly or slowly it had been warmed. "Adaptation" was completely effective only for the temperature of the water in which the hand was adapted, or for higher temperatures. Thus, if a hand adapted in water at $10^{\circ} \mathrm{C}$. were withdrawn and plunged directly into water at $5^{\circ} \mathrm{C}$. the cycle of pain would occur as usual, the onset being somewhat delayed and the intensity of pain somewhat less than it would have been had a warm hand been immersed.

\section{Spatial summation}

When applied to a sensation, the term "spatial summation " means that the larger the area stimulated, the weaker would be the stimulus necessary to produce the sensation in question. Hardy and Oppel (16) demonstrated spatial summation for heat and cold sensations on the forehead. That is, as the area exposed to the heat radiation was increased, the strength of stimulus necessary to evoke sensation decreased almost in proportion. Hardy, Wolff and Goodell (17) found that pain induced by radiant heat on the forehead did not have this property of spatial summation. With the water bath at $18^{\circ} \mathrm{C}$., as already pointed out, barely detectable pain occurs in an immersed hand. When one finger was immersed, pain of the same intensity occurred. Immersing one finger at $0^{\circ} \mathrm{C}$. brought on pain of intensity equal to that experi- enced when the whole hand was immersed. This type of pain, then, like burning pain, fails to show the effect of spatial summation.

\section{III. "Cold Pain" in parts other than the hand}

The hand is not the only part of the body in which pain can be induced by local exposure to cold. The feet and legs were similarly sensitive to cold, as was the face, tongue, scrotum, etc. When the vertex of the head was dipped in cold water, pain was induced in the vertex following the pain cycle described for the hand. The sensation spread down the back of the head and through the temples, and appeared to be more intense than that experienced for the hand under like conditions. The highest temperature at which the headache could be induced, however, was $18^{\circ} \mathrm{C}$.

In two sites on the body it was found that pain could not be induced in our subjects by cold water. These were the lobe of the ear and the glans penis. The explanation for these exceptions is not clear at present.

\section{$I V$. The behavior of skin temperature}

The skin temperature fell off sharply upon immersion. The rate of cooling of the skin was rapid for the first minute. By this time 90 to 95 per cent of the total fall in skin temperature had occurred and cooling continued at an increasingly slower rate (Figure 4).

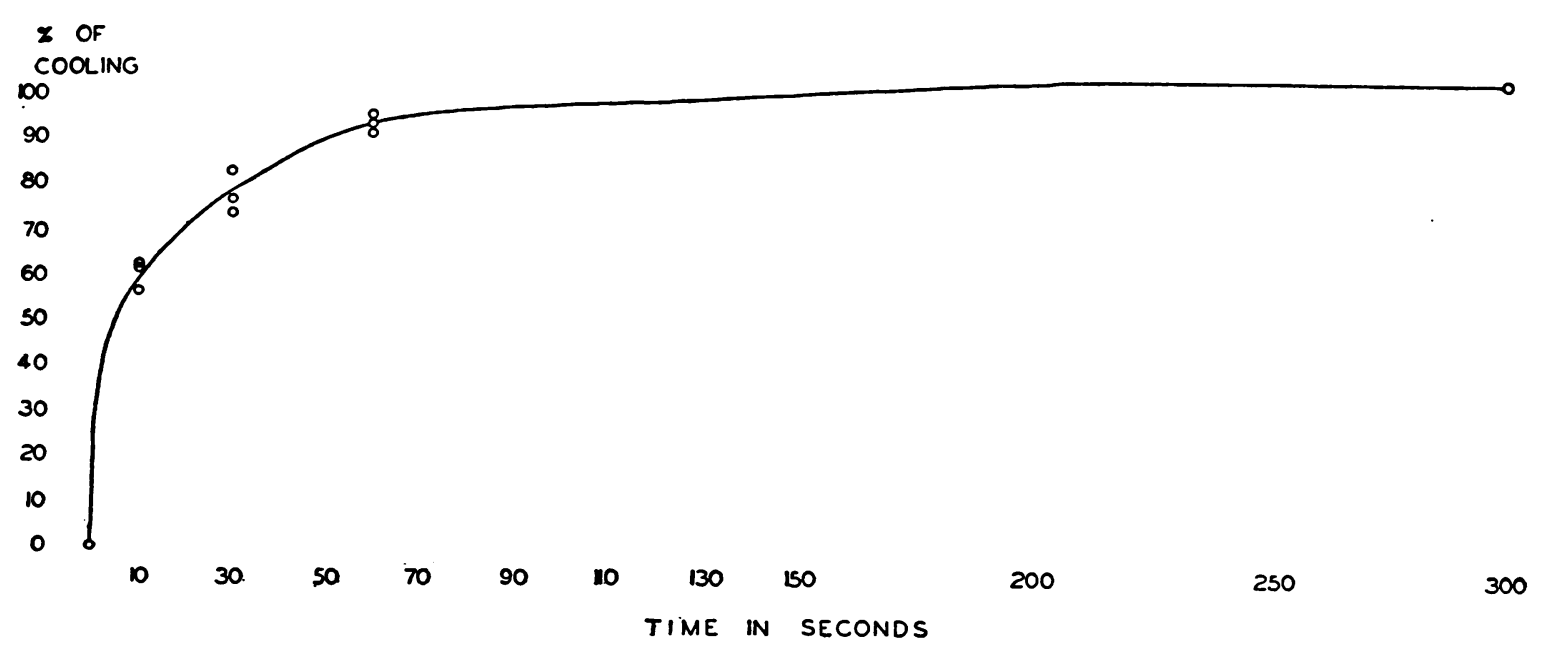

Fig. 4. The Course of Skin Temperature Showing the Rate of Cooling at Various Bath Temperatures 


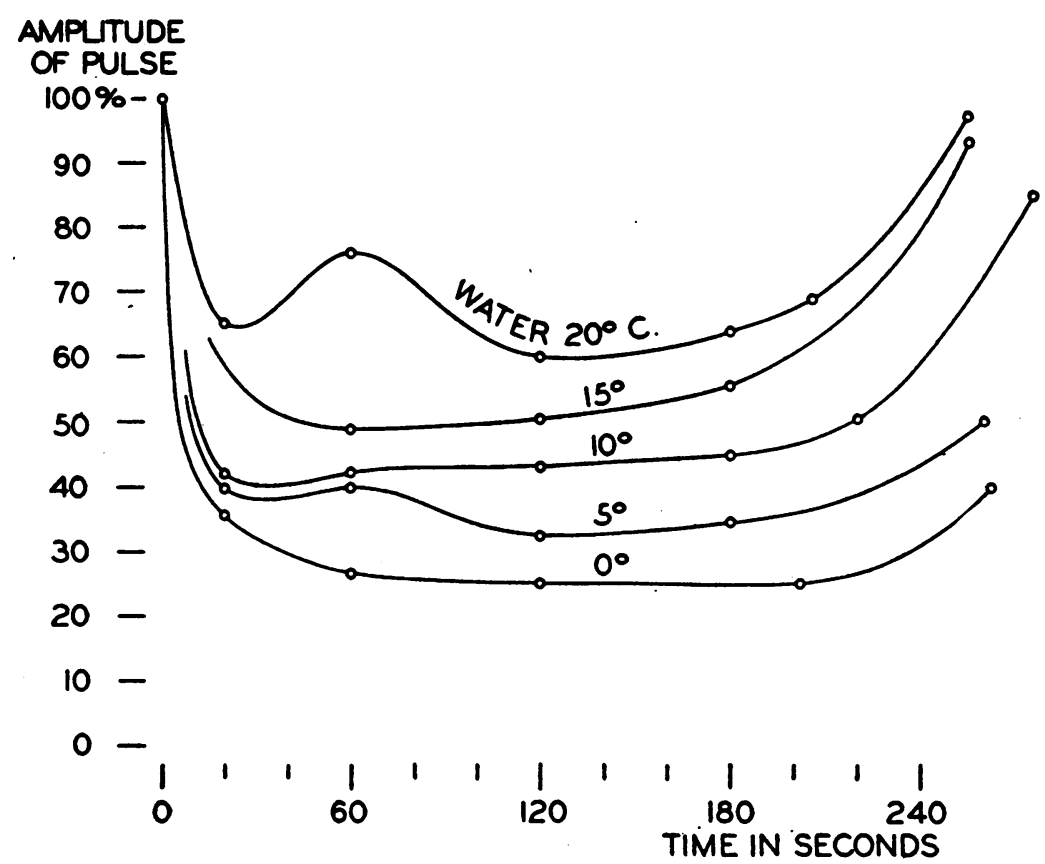

Fig. 5. The Course of Skin Temperature and Amplitude of Pulsation of the Digital Artery With the Left Hand Immersed in Water at VarIOUS TEMPERATURES

\section{$V$. Behavior of the digital pulse}

The amplitude of the digital pulse decreased suddenly upon immersion of the hand (Figure 5), reaching its minimum at the time when pain in the hand was at a maximum. Following this there was a gradual return of the amplitude toward normal. This effect was most evident at temperatures of $9^{\circ} \mathrm{C}$. or higher. Figure 6 illustrates the relationship between pain, "pins and needles" sensation and the amplitude of pulsation of the digital artery, with the hand immersed at $9.5^{\circ} \mathrm{C}$. The pulsations began to decrease in amplitude directly after immersion of the hand. The pulse waves of minimal amplitude correspond in point of time to the maximum intensity of pain in the hand. As the pain intensity began to decrease and the "pins and needles" sensation appeared, the amplitude of pulsation of the digital artery increased again, reaching its former level after the hand had completely adapted to the "pins and needles" sensation. At temperatures below $10^{\circ} \mathrm{C}$. the intense cold usually appeared to hold the arteries more or less tightly constricted, thus masking the vasodilator effect apparent at higher temperatures (Figure 5).
Figure 7 illustrates a simultaneous recording of pulsations in the immersed fingers and the fingers of the opposite hand. The sharp decrease in amplitude of pulsation of the digital artery in the painful hand was closely paralleled by the tracing of the opposite hand. In the latter, however, there occurred shortly a vasodilator effect which brought the amplitude of pulsations back to normal.

\section{Behavior of blood pressure}

Ten to 60 seconds after immersion of the hand in cold water, and approximately coincident with the onset of pain, a sharp rise in blood pressure occurred. This reached its maximum at about the point of maximum pain. With the onset of "adaptation" the level of blood pressure declined slowly and returned to normal after the pain and "pins and needles" sensation had disappeared. When the adapted hand was withdrawn and promptly re-immersed, there was no recurrence of blood pressure elevation just as there was no recurrence of pain. Like pain, the blood pressure elevation bore a direct relationship to the degree of local cooling (Figure 8). No elevation of blood pressure occurred in water warmer than $18^{\circ} \mathrm{C}$. 

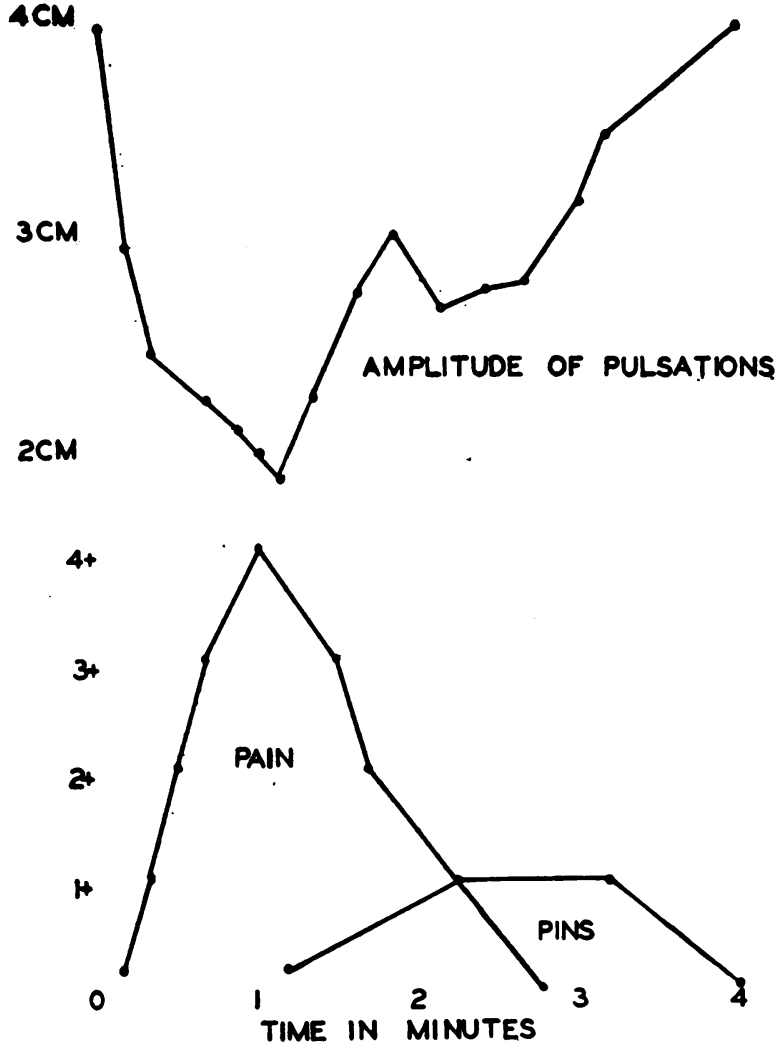

Fig. 6. Relationship of the Amplitude of Pulsations of the Digital Artery to the Occurrence of Pain and "Pins and Needles" Sensation at $9.3^{\circ} \mathrm{C}$.

The return to normal amplitude of pulsation was unusually rapid and complete in this record.

\section{The effect of ischemia}

Using a sphygmomanometer cuff maintained at a pressure of $200 \mathrm{~mm}$. of mercury, the blood supply to the arm was interrupted for 10 seconds, 5 minutes, 15 minutes, 35 minutes, and 45 minutes before the hand was immersed. The inflated cuff was left in place during the period of immersion while observations were recorded. The pain and "pins and needles" sensation occurred at their usual times, both with somewhat diminished intensity. "Adaptation" took place as usual. In the experiment in which the circulation was cut off for 45 minutes, it was noted that touch sensation, cold sensation and pain to pin prick had gone entirely from the hand before immersion (14) but, in spite of this, "cold pain" occurred as usual when the hand was immersed.

Comment. It is known that, when anesthesia is induced by interrupting the blood supply to a part, the large fibers of class $A$ are the first which cease to conduct impulses and that, as the ischemia is continued, nerve conduction stops in progressively smaller fibers until the smallest non-myelinated fibers of class $C$ are the last to drop out (18). Cocaine anesthesia, on the other hand, has been shown to affect nerve fibers in just the opposite sequence (19), namely, the small class $C$ fibers are the first ones blocked and the largest fibers of class A the last. Accordingly, since "cold pain" from the above experiment in which the area was rendered ischemic appeared to be conducted along the smallest fibers of the nerves, it was determined to confirm this observation by testing the effect of procaine anesthesia.

\section{Effect of procaine}

Three cubic centimeters of a 1 per cent solution of procaine hydrochloride were injected around the left ulnar nerve, and 30 seconds after the injection was completed the fourth and fifth fingers of the left hand were immersed in water at $10^{\circ} \mathrm{C}$. Scarcely any "cold pain" appeared. Nevertheless, all other sensory modalities, including pain to pinching and pin prick, were intact at this time. Later, an incomplete and transient anesthesia developed in the ulnar distribution, but it had been possible first to eliminate "cold pain" while pin prick and deep pressure pain were still appreciated.

\section{Effect of sympathectomy}

That this modality of "cold pain" is mediated by small non-myelinated fibers of class $C$ appeared to be indicated from these data. Other fibers of similar size are the sympathetic vasomotor fibers. In order to determine any possible influence of these upon the phenomenon of "cold pain ", a patient who had had the cervical sympathetic ganglia on the right removed within the previous four weeks for intractable asthma was used as a subject. It was found that his right hand was markedly warmer than his left. The completeness of sympathectomy was demonstrated by comparing the change in skin resistance in the two hands upon the application of a nociceptive stimulus. Repeatedly there was a marked change in resistance on the left and none at all on the right. When the left arm was immersed in cold water, the usual cycle of pain and blood pressure eleva- 


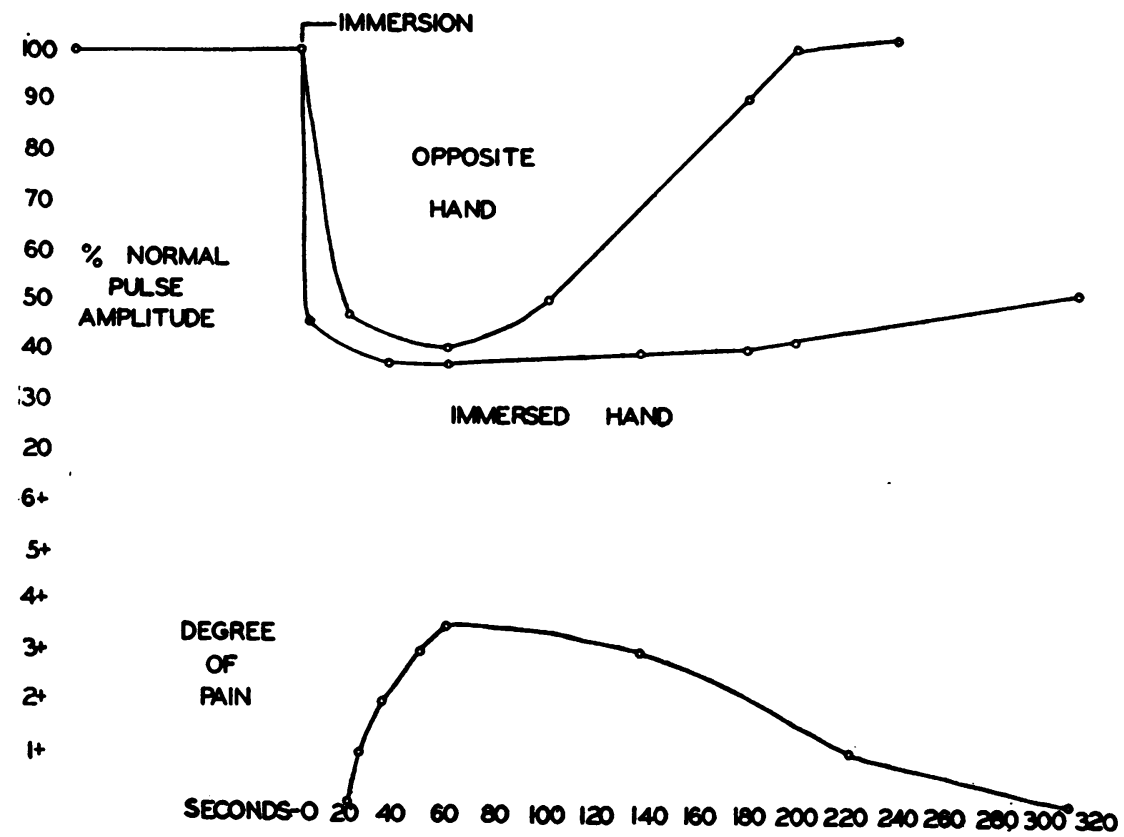

Fig. 7. A Comparison of the Amplitude of Pulsation of the Digital Artery in the Immersed Hand With That of the Opposite Hand

\section{DIASTOLIC}

\section{B.P. RISE_}

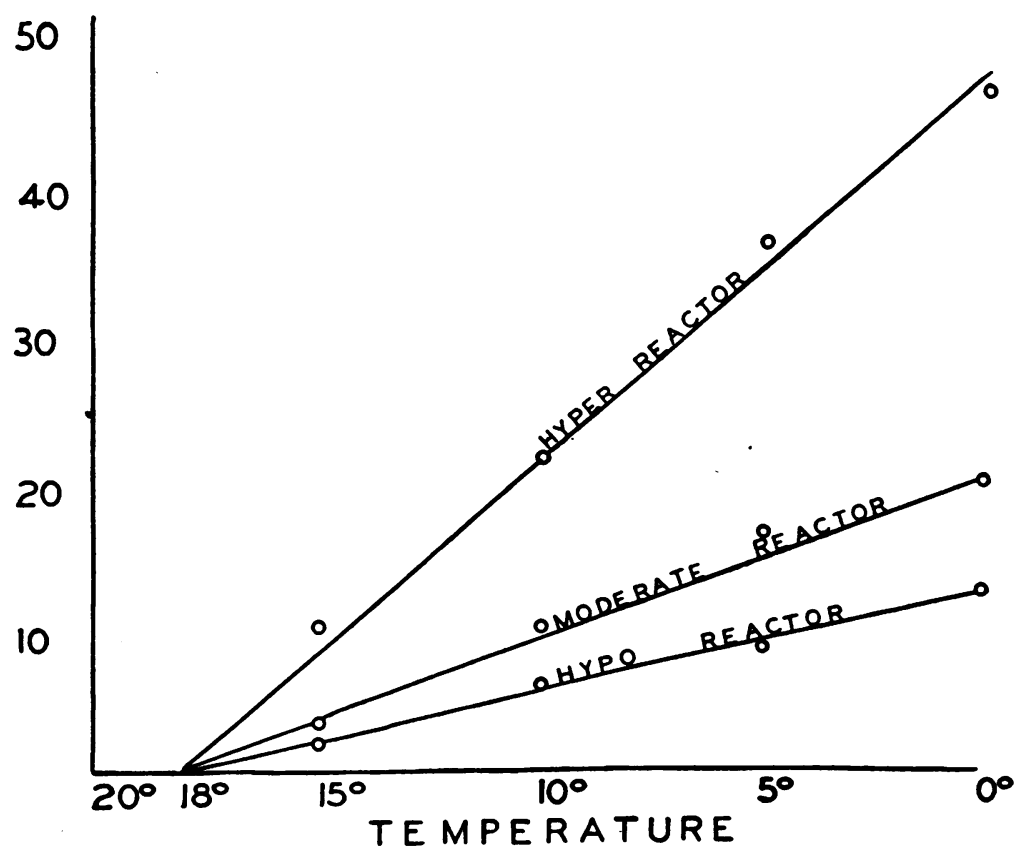

Fig. 8. Relationship Between the Blood Pressure Elevation and the Strength of Cold Stimulus in Different Individuals at Various TEMPERATURES 
tion occurred. When the right hand was immersed, however, the pain was much more severe and the elevation of blood pressure much more marked. At $10^{\circ} \mathrm{C}$., for example, there was a diastolic rise of $10 \mathrm{~mm}$. of $\mathrm{Hg}$ when the left arm was immersed and $20 \mathrm{~mm}$. of $\mathrm{Hg}$ when the right arm was immersed. When the circulation to the arm was interrupted by a sphygmomanometer cuff, the effects were not altered.

\section{$X$. The effect of slow cooling}

If the hand were immersed in water at $20^{\circ} \mathrm{C}$., a temperature at which no pain could be induced, and the temperature of the water slowly lowered by successive steps to $0^{\circ} \mathrm{C}$. (over a period of more than 60 minutes), no pain was felt. The sensation of "pins and needles", however, occurred at about $12^{\circ} \mathrm{C}$. whether the cooling was slow or rapid, and became more intense with further cooling, adapting slowly at each new temperature. The actual intensity of "pins and needles" sensation was not as great with slow cooling as it was at comparable temperatures when the hand was cooled rapidly. The slower the cooling, the less marked was the sensation, and it is quite possible that if the hand were cooled slowly enough the sensation would not appear at all.

\section{$X I$. The effect of successive cooling of parts}

One hand was plunged into cold water where it was allowed to remain until complete "adaptation" had taken place. Then the other hand was immersed. The usual cycle of pain occurred in the freshly immersed hand without any pain occurring in the already adapted hand. After the second hand was thoroughly adapted, one forearm was introduced. Again pain was felt in the newly immersed part but not in the hands. Similarly, after submerging the other forearm, only that part experienced pain while the other members were " adapted".

\section{$X I I$. The effect on "cold pain" of agents which alter the contractile state of arteries}

$A$. The effect of vasodilator agents. The contents of an ampule of amyl nitrite was inhaled on two occasions while the hand was immersed in cold water. There was no detectable effect on the pain. One milligram of histamine injected subcutane- ously was similarly unavailing in modifying the pain caused by cold, although the subject became flushed as a result of peripheral dilatation.

$B$. The effect of vasoconstrictor agents. Pitressin, 1 cc., was given hypodermically to 3 subjects to determine the effect of strong vasoconstriction on the intensity and duration of "cold pain". Readings were made 15 minutes, 45 minutes, $11 / 2$ hours and 2 hours after injection. It was found that both intensity and duration of pain were increased by 50 per cent. The maximum effect was noted at $\mathbf{4 5}$ minutes. After 2 hours the effect had disappeared.

The fact that pitressin brought about an increase in intensity and duration of "cold pain" suggests that vasospasm may be a factor in the production of " cold pain ". Ray and Wolff (20), however, in experiments on the pain sensitivity of cranial arteries, brought about a brisk vasoconstriction by painting epinephrine directly on a cranial artery without inducing pain. Yet an epinephrine pack placed in the nose is known to cause intense pain. We found that this pain could be greatly accentuated by breathing cold air and nearly completely relieved by breathing warm air. It may be that here one is dealing with a pain arising from vasoconstriction which is relieved by the vasodilator influence of warmth.

In order to test this possibility further, it was determined to induce a powerful vasoconstriction in a hand still immersed in cold water but already adapted to "cold pain". Accordingly, the left hands of the subjects were immersed in water at $10^{\circ} \mathrm{C}$. and, after "adaptation" was complete and the blood pressure had returned to its control level, 0.00025 gram epinephrine was injected rapidly intravenously. Almost at once there was a sudden and dramatic rise in the systolic blood pressure to more than $200 \mathrm{~mm}$. Hg. At this time the subjects were pale, weak, and dyspneic, with accelerated pulse and respirations. The mouth was dry with an unpleasant, faintly metallic taste in it, and soon a moderately severe centrally placed headache appeared. The adapted hand in the water at first tingled but there was no "cold pain"; after the blood pressure had passed its peak, the tingling was replaced by "cold pain". The latter persisted until the blood pressure had fallen nearly to normal. Then the pain gave way to "pins and needles", as usual, and finally, as the blood pres- 


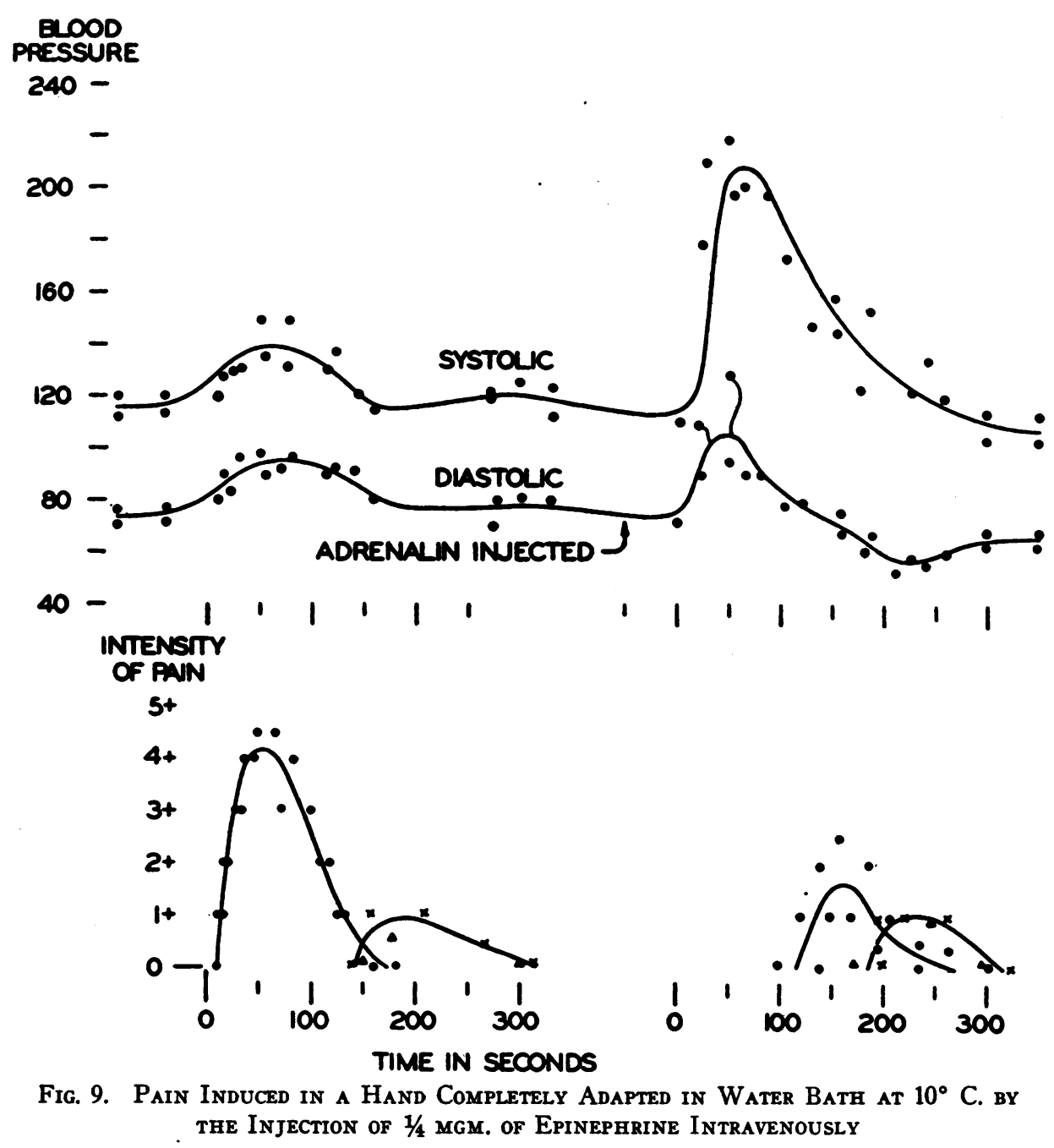

sure returned to normal, that sensation ceased (Figure 9).

Comment. Having been able to reproduce pain similar to "cold pain" in this way, one is still not entirely clear as to whether or not this effect was brought about by vasoconstriction or by some other effect of epinephrine. The effects of epinephrine upon deep-lying blood vessels are not entirely agreed upon by investigators, and it is quite possible that the drug effected a warming of the deeper structures of the hand and abolished "adaptation" by increasing the thermal gradient, thus again rendering the hand susceptible to "cold pain" from the water in which it was immersed.

\section{DISCUSSION}

From the above data what inferences can be made about the aching pain described, and to what process can we attribute the phenomenon of "adaptation" to this pain?

Undoubtedly, the degree of pain experienced is closely allied to the strength of the cold stimulus, since the intensity, as well as the total amount of pain felt, increases in direct proportion to the lowering of the temperature of the water bath in which the hand is immersed. The first question to be answered is whether or not the pain thus experienced depends upon extreme stimulation of cold sensation (21, 22, 23, 24). Evidence upon this point is complete. As soon as the subject's hand is immersed he feels cold but no pain. The cold sensation continues throughout the cycle of pain and persists unaltered long after the pain is gone. In the experiment in which ischemia of the nerve was obtained by occluding the blood supply to the arm for 45 minutes, cold sensation 
in the hand was abolished. "Cold pain", however, could be induced as usual. Clearly, the pain is not secondary to stimulation of the sense of cold.

Since separate pain fibers mediate the sensation of "cold pain" it is desirable to know their character. The experiments on partial block of the nerve trunks by ischemia, on the one hand, and procaine, on the other, suggest that the fibers in question are the small, non-myelinated ones of class $\mathrm{C}$.

It is clear that purely local changes in the immersed part are responsible for initiating the pain, as evidenced from the distribution of the sensation as well as from the fact that its behavior is not altered by interruption of the blood supply and most of the nervous impulses going to the part. Furthermore, the purely local character of the phenomenon was demonstrated in the experiments in which parts were immersed successively into the water bath.

The fact that the intensity of pain experienced parallels closely the degree of cold, and the finding that "adaptation" is effective only while the hand and water temperature difference is small, have already been emphasized. These data suggest that the thermal gradient in the hand is responsible for "cold pain" and that, when the gradient becomes less after the first minute of cooling, "adaptation" occurs because the stimulus for pain is abolished. It seems unlikely that the site of production of this pain stimulus is in the skin since the character of the pain is not that of any other known cutaneous pains. These are invariably bright and burning in quality, while "cold pain" is deep and aching. The deeper tissues and blood vessels are the other possible sites where "cold pain" may be initiated.

Certain findings suggest that the blood vessels may be concerned in the production of "cold pain ". There is a marked vasoconstriction associated with the occurrence of "cold pain" which is reflected in the tracings of the amplitude of pulsations of the digital artery. With the onset of "adaptation", there appears to be a relaxation of the vasospasm and an increase in the amplitude of the digital pulse toward the control level.

Several investigators have studied blood vessels with reference to their pain sensitivity $(25,26,27$, 28). The experiments on humans by Ray and
Wolff (20) show clearly that cranial arteries are pain-sensitive to faradic and mechanical stimulation. If the vessels were stretched, distended from within or without, pinched or pulled, very definite pain resulted.

If pain results from vasoconstriction, then it must be a purely local phenomenon. What is the relationship of the reflex vasomotor activity with its resultant hypertension, however, to the other events which occur as a result of plunging a hand into cold water? Could it represent simply a reaction to the pain experience? It is known that pain applied anywhere on the body induces hypertension and, as we have emphasized repeatedly, the height of blood pressure rise obtained corresponds closely to the intensity of pain experienced, which in turn depends upon the degree of cooling. Hines and Brown (1) separate subjects into three groups according to their blood pressure rise in the "cold pressor" test. They name the categories-Hyperreactors, Normal Reactors, and Hyporeactors. They look upon the Hyperreactors as potential hypertensives. Whether or not this inference is justifiable, it is quite clear that subjects do react differently to local cooling in terms of blood pressure rise. Moreover, they react according to a definite pattern. In a given individual, whether a hyper-, hypo-, or normal reactor, we found that the level to which the blood pressure rose increased in proportion to the lowering of the temperature of the water bath (Figure 8).

The vasopressor effect seems clearly to be influenced by the pain. In the experiment in which all the other sensory modalities were blocked by ischemia of the nerve, the elevation of blood pressure occurred as usual with "cold pain". Analgesic drugs of various sorts-aspirin, codeine and alcohol-lowered the pressor effect as they reduced the pain. In the subject who had had a recent cervical sympathectomy on the right, we found that much more intense pain was experienced when the sympathectomized hand was plunged into the cold water than when the normal hand was immersed. In other respects, sensory examination of the two hands was identical. A much greater blood pressure elevation was associated with the more painful experience, however.

It appears that the pressor effect in these experiments is related either to the pain itself or to 
the subject's reaction to the pain. Several facts indicate that the latter possibility is the more likely. Schumacher and others (29) have shown that, while reaction to pain is by no means constant from one individual to another, the pain threshold of a large group of individuals is practically identical. This suggests but does not prove that the intensity of "cold pain" experienced by all subjects is about the same. The differences in blood pressure, then, must be due to differences in reaction to the pain from one subject to another. Some confirmation of this notion was obtained from an experiment in which a Hyperreactor was given 0.26 gram of sodium pentobarbital hypodermically in divided doses one hour apart. Onehalf hour after the second dose his hand was placed in water at $0^{\circ} \mathrm{C}$. and his blood pressure response was that of a Hyporeactor. It has been shown that barbiturates in therapeutic dosage have relatively little effect on the pain threshold. The change must have been due to the effect of the drug on the subject's reaction to pain. Therefore, the term "Hyperreactor" of Hines and Brown appears to be a particularly appropriate one. The fact that this group reacts in such a way to a distressing stimulus which is equal in intensity to that to which others are exposed who react less strongly may well shed light upon this subsequent development of a permanent hypertension. These findings seem to support the thesis of Hines and Brown (1). ${ }^{1}$ Whether or not the blood vessels are concerned in the mechanism of "cold pain" cannot be decided from the evidence in hand. There is, however, much to suggest that they are.

\section{SUM MARY}

A deep, aching, painful sensation induced by immersing a part of the body in cold water is described. The behavior of this phenomenon is found to follow a definite pattern, namely, that regardless of strength of stimulus, pain reaches its maximum in approximately 60 seconds. Thereafter, the pain gradually subsides, giving way to

\footnotetext{
1 There is one important modification which should be introduced into the technic of the "cold pressor" test. If the thermal stimulus is to be compared from one test to another, it is essential that the water in which the hand is immersed be stirred vigorously in order to avoid a warm zone in the immediate vicinity of the immersed hand.
}

a sensation of "pins and needles" which soon, in turn, is terminated. The intensity of the pain and the total amount of pain were found to depend directly upon the degree of cooling, with "adaptation" occurring when the difference between the hand and water temperature was small. Warming the hand or lowering the bath temperature abolished the protective effect of "adaptation". The mechanism whereby the pain is produced and the reason for its "adaptation" were investigated. It was found that:

1. This pain is entirely separate from and independent of the sensation of cold itself.

2. This type of pain does not show the phenomenon of spatial summation since exposure of one finger to cold causes pain of equal intensity to that experienced when the whole hand is so exposed.

3. The pain may be induced on nearly all parts of the body. In each case the highest bath temperature at which pain can be obtained is $18^{\circ} \mathrm{C}$.

4. The skin temperature of the immersed part decreases rapidly for the first minute after immersion and then much more slowly.

5. The amplitude of pulsation of the digital artery parallels inversely the intensity of "cold pain" experienced.

6. The blood pressure-raising effect is proportional both to the intensity of pain experienced and to the degree of cold.

7. Ischemia, which is known to produce pain in muscular structures, is not a significant factor in the production of " cold pain".

8. By selective partial block of nerve trucks, it was indicated that "cold pain" is carried by the small, non-myelinated fibers of class $C$.

9. Sympathectomy was found to augment the intensity of pain derived from cold.

10. It was found possible to lower the bath temperature to zero without pain being felt in the immersed hand if the cooling were carried out slowly enough.

11. By successively cooling different parts it was learned that the production of "cold pain" depends on local changes in the part cooled.

12. Evidence is presented which indicates that the elevation of blood pressure which results from immersion of a part in cold water is a measure of the subject's reaction to "cold pain". 


\section{CONCLUSIONS}

Pain due to local cooling is altogether separate from the sensation of cold itself. It is apparently mediated through small, non-myelinated fibers of class C. Its intensity, however, depends directly upon the degree of cooling. The stimulus required for the production of "cold pain" may be found in the thermal gradient in the tissues of the immersed hand. It is possible that this stimulus brings about a painful vasospasm in the part. Relaxation of this local vasospasm may occur as the thermal gradient is decreased, thus accounting for "adaptation". It appears that the "cold pressor" effect is a measure of reaction to pain.

\section{BIBLIOGRAPHY}

1. Hines, E. A., and Brown, G. E., A standard stimulus for measuring vasomotor reactions: Its application in the study of hypertension. Proc. Staff Meeting Mayo Clinic, 1932, 7, 332.

2. Pickering, G. W., and Kissin, M., The effects of adrenaline and of cold on the blood pressure in human hypertension. Clin. Sc., 1936, 2, 201.

3. Lewis, T., Vascular reaction to cold. Heart, 1930, 15, 177.

4. Edes, B., and Dallenbach, K. M., The adaptation of pain aroused by cold. Am. J. Psychol., 1936, 48, 307.

5. Martin, S. J., Marcellus, F. S., and Sykowski, P., Plethysmographic studies with special reference to waves of respiration. J. Lab. and Clin. Med., 1938, 24, 111.

6. Bolton, B., Carmichael, E. A., and Stürup, G., Vasoconstriction following deep inspiration. J. Physiol., 1936, 86, 83.

7. Turner, R. H., Burch, G. E., and Sodeman, W. A., Studies in the physiology of blood vessels in man. III. Some effects of raising and lowering the arm upon the pulse volume and blood volume of the human finger tip in health and in certain diseases of the blood vessels. J. Clin. Invest., 1937, 16, 789.

8. Hermann, H., Morin, G., and Cier, J., Réactions vasculaires locales consécutives au refroidissement de la peau chez le chien. Comptes Rendues Soc. de Biol., 1937, 126, 1019.

9. Lewis, T., Supplementary notes upon the reactions of the vessels of the human skin to cold. Heart, 1931, 15, 351.

10. Lewis, T., and Pickering, G. W., Vasodilation in the limbs in response to warming the body; with evidence for sympathetic vasodilator nerves in man. Heart, 1931, 16, 33.

11. Lewis, T., The Blood Vessels of the Human Skin and Their Responses. Shaw, London, 1927.
12. Wilkins, R. W., Doupe, V., and Newman, H. W., The rate of blood flow in normal fingers. Clin. Sc., 1938, 3, 403.

13. Goldschmidt, S., and Light, A. B., Effect of local temperature upon peripheral circulation and metabolism of tissues as revealed by the gaseous content of venous blood. Am. J. Physiol., 1925, 73, 146.

14. Herget, C. M., Granath, L. P., and Hardy, J. D., Studies in temperature sensation. V. Thermal discrimination and the Weber-Fechner Law. (In Press.)

15. Herget, C. M., and Hardy, J. D., Studies in temperature sensation. VII. Spatial summation of heat. (In Press.)

16. Hardy, J. D., and Oppel, T. W., Studies in temperature sensation. III. The sensitivity of the body to heat and the spatial summation of the end organ responses. J. Clin. Invest., 1937, 16, 533.

17. Hardy, J. D., Wolff, H. G., and Goodell, H., Studies on pain. A new method for measuring pain threshold: Observations on spatial summation of pain. J. Clin. Invest., 1940, 19, 649.

18. Lewis, T., and Pochin, E. E., Effects of asphyxia and pressure on sensory nerves of man. Clin. Sc., 1937, $3,141$.

19. Gasser, H. S., and Erlanger, J., The rôle of fiber size in the establishment of a nerve block by pressure or cocaine. Am. J. Physiol., 1929, 88, 581.

20. Ray, B. S., and Wolff, H. G., Experimental studies on headache; pain-sensitive structures of the head and their significance in headache. Arch. Surg., 1940, 41, 813.

21. Erb, W., Krankheiten der peripheren-cerebrospinalen nerven. 1876, Vogel, Leipzig.

22. Schiff, M., Lehrbuch der Physiologie. Vol. 1. Muskel- und nervenphysiologie. Schauenburg, Lahr, 1858-59.

23. Goldscheider, A., Gesammelte Abhandlungen. Barth, Leipzig, 1898.

24. Von Frey, M., Die Gefühle und ihr Verhältnis zu den Empfindungen. Beit. z. Physiol. des Schmerzsinnes. Berichte über die verhandlung d. königl. sächs. Gesellshaft d. Wissenschaften, Leipsig, 1894.

25. Woolard, H. H., Weddell, G., and Harpman, J. A., Observations on the neurohistological basis of cutaneous pain. J. Anat., 1940, 74, 27.

26. Livingston, $\mathrm{W}$. K., The blood vessels as a possible source of visceral pain. Am. Heart J., 1930, 5, 559.

27. Burget, G. E., and Livingston, W. K., Pathway for visceral afferent impulses from the fore-limb of the dog. Am. J. Physiol., 1931, 97, 249.

28. Moore, R. M., and Moore, R. E., Studies on the painsensibility of arteries. I. Some observations on the pain-sensibility of arteries. Am. J. Physiol., 1933, 104, 259.

29. Schumacher, G. A., and others, Uniformity of the pain threshold in man. Science, 1940, 92, 110. 